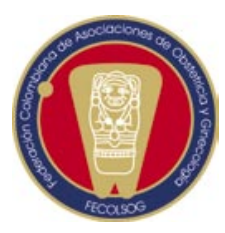

ARtículo de ReVISIÓN

\title{
MANEJO DE LA INFECCIÓN MATERNA CON VIH Y DEL RECIÉN NACIDO EXPUESTO*
}

\author{
Managing HIIV during pregnancy and in exposed newborn children \\ Carlos Eduardo Fonseca, M.D.**, Franklyn Edwin Prieto, M.D., MSc.***
}

Recibido: septiembre 8/2004 - Revisado: septiembre 28/2004 - Aceptado: marzo 28/05

\section{RESUMEN}

El VIH/SIDA afecta cada vez más a las mujeres, lo que aumenta la posibilidad de transmisión perinatal. La detección temprana del VIH mediante el acceso a la consejería y prueba voluntaria durante el control prenatal facilitan el tratamiento anti-retroviral oportuno que disminuye la transmisión durante el embarazo y el parto, y en el puerperio, asociado a la lactancia materna. En la presente revisión se condensan los principales aspectos del manejo de la infección materna con VIH y del recién nacido expuesto. La aplicación de un protocolo integral de atención facilita la disminución de la transmisión a niveles menores de $2 \%$.

Palabras clave: virus de inmunodeficiencia humana (VHI), transmisión de la enfermedad, control y prevención/vertical, atención prenatal, diagnóstico prenatal, terapia antiretroviral altamente activa.

\section{SUMMARY}

HIV/AIDS attacks women progressively; in turn this raises the risk of HIV mother-to-child transmission. Early HIV diagnosis, through HIV counselling and voluntary testing during antenatal check-ups, enables opportune anti-retroviral treatment; it could

* Nota del editor: dada la importancia del tema y como una excepción a la regla, se decidió publicar este artículo con la debida autorización de la revista de la Sociedad Colombiana de Pediatría, puesto que es una actualización del artículo "SIDA PERINATAL" publicado en esa revista en el volumen 38 (2) de marzo de 2003.

** Docente de pediatría, Universidad Surcolombiana. Neiva, Huila, Colombia. Correo electrónico: cefonseca2000@yahoo.com.

*** Coordinador Nacional, Proyecto Nacional de la Reducción de la Transmisión Madre Hijo de VIH. Bogotá DC, Colombia.

Correo electrónico: franklynprieto@yahoo.com. diminish antepartum, intrapartum and postpartum HIV transmission. The main topics dealt with in this review concern managing HIV in pregnancy and exposed newborn children. An integral health-care approach should ensure reducing mother-to-child transmission risk to less than $2 \%$.

Key words (MeSH): human immunodeficiency virus (HIV); disease transmission, vertical/prevention and control; prenatal care; prenatal diagnosis; highly active antiretroviral therapy.

\section{INTRODUCCIÓN}

$$
\begin{aligned}
& \text { Una madre necesita un bebé no infectado } \\
& \text { y el bebé necesita una madre saludable. }
\end{aligned}
$$

Hoosen Coovadia

El Programa Conjunto de la Naciones Unidas sobre el VIH/SIDA (ONUSIDA) estableció que había, a julio de 2004, 37,8 (rango de 34,6-42,3) millones de personas viviendo con VIH/SIDA, de las cuales 17 millones $(15,8-18,8)$ son mujeres; 18,7 millones, hombres y 2,1 millones (1,9-2,5), menores de 15 años; en el 2003 se tendrían 4,8 millones de nuevas infecciones. El 67\% de los afectados habita en África y 5,4\% en América Latina y el Caribe. ${ }^{1}$ De la población mundial de 15 a 49 años, el 1,1\% están infectados con VIH. ${ }^{2}$

Desde el inicio de la epidemia de VIH/SIDA han muerto 20 millones de personas. ${ }^{1}$ Para Latinoamérica se calcula que 1,6 millones $(1,2-2,1)$ de personas viven con VIH, cada año se pre- 
sentan 200.000 nuevos casos y 84.000 muertes por SIDA. ${ }^{2}$

La mayoría de los casos en niños se debe a infección vertical; con una estimación de 630 (570-740) mil niños menores de 15 años infectados anualmente ${ }^{1}$. En el 2003 había 2,1 millones de niños menores de 15 años viviendo con VIH; en este mismo año murieron 490 (440-580) mil niños, 90\% en África.

En Latinoamérica, el número de casos acumulados de VIH/SIDA de Colombia es superado por Brasil y México. La prevalencia en nuestro país se ha calculado entre 0,6 y $0,7 \%$, con una incidencia de $0,06 \%$. El comportamiento de la epidemia de VIH/SIDA en Colombia es de transición, del predominio de casos en hombres que tienen sexo con otros hombres a la afectación de la población heterosexual femenina y masculina; ${ }^{4}$ de una razón hombre:mujer de 12:1 en 1998, alcanza 3:1 en 2004. Esta progresión ha sido evidente en algunas regiones como la Costa Atlántica donde la relación llega a $3: 1{ }^{3}$

A medida que el VIH/SIDA afecta a la población femenina, aumenta el número de casos de mujeres embarazadas infectadas, ${ }^{3}$ que al no ser detectadas a tiempo, transmitirán la infección a su hijo o hija.

La transmisión del VIH al niño sucede por tres mecanismos diferentes: ${ }^{5,6}$

1. Transmisión intra-parto. Representa entre 40 y $80 \%$ de los casos y es causada por el contacto fetal con la sangre y el líquido amniótico.

2. Transmisión in útero. Ocasionalmente se ha identificado VIH en el tejido fetal a la octava semana de gestación. Es responsable del 10\% al 25\% de los casos.

3. Transmisión a través de la leche materna. La lactancia materna es el mecanismo de transmisión en $14 \%$ a $30 \%$ de los casos.

La provisión de cuidados prenatales y atención integral para VIH, que incluye el acceso a la terapia anti-retroviral actual, es difícil y costosa. Esta provisión necesita de la coordinación de los servicios de salud pública y clínicos (obstétricos y pediátricos); monitoreo intensivo de la replicación viral, y consejería, educación y soporte social. Un tratamiento óptimo e intensivo para la infección materna con VIH puede reducir la probabilidad de transmisión madrehijo de VIH. Éste comprende la adecuada decisión de la vía del parto, profilaxis con ZDV y suministro de leche de fórmula.

El nacimiento de un niño infectado con VIH es una señal de oportunidades perdidas, y ocasionalmente, de falla profiláctica. Una oportunidad perdida se da por el desconocimiento de la gestante sobre su estado serológico ante el VIH o de su riesgo de adquirirlo. También refleja que las gestantes no acceden al control prenatal, que no se les ofreció o realizó la prueba, que no recibieron oportunamente tratamiento y profilaxis, que no hubo adherencia al tratamiento. $^{7}$

A continuación se hace una revisión de los aspectos principales de la atención de la mujer embarazada infectada con VIH y su hijo o hija expuesto, resaltando los aspectos más relevantes de dicha atención.

\section{ACCESO AL DIAGNÓSTICO ${ }^{8}$}

Durante el control prenatal, las mujeres embarazadas deben recibir asesoría y consejería en VIH y en el caso de aceptar mediante consentimiento informado, también debe realizarse una prueba diagnóstica para VIH, que incluye la prueba de tamizaje con Elisa y la confirmación con Western Blot. Por esta razón, la demanda inducida del control prenatal es una prioridad en la acción de salud pública.

Entre las razones que justifican el realizar el tamizaje de VIH en gestantes se tiene el que permite una evaluación de riesgo, brinda un reforzamiento para la reducción de comportamientos de riesgo relacionados con VIH, permite la remisión a los servicios de salud, la realización de un diagnóstico y tratamiento oportuno, el suministro de información sobre decisiones en salud sexual y reproductiva, la prevención de la transmisión a otros, el soporte psico-social, la reducción de la transmisión madre-hijo de VIH (incluyendo riesgo de transmisión, forma de prevenir transmisión y el pronóstico de los niños infectados con VIH). ${ }^{9}$

En el caso que la gestante tenga una prueba Elisa no reactiva o Western Blot negativa se considerará 
como no infectada con VIH, excepto que haya exposición previa o durante el embarazo a una pareja infectada con VIH, situación en la que se recomienda repetir la prueba a los 2-3 meses.

Deben proveerse todos los mecanismos para prevenir la transmisión de la infección durante la gestación, el parto y el puerperio. Incluso, ante la dificultad para la confirmación del diagnóstico, la gestante con un resultado indeterminado en la prueba de Western Blot podría considerarse positiva para recibir el tratamiento. Los casos indeterminados pueden ser causados por una respuesta incompleta de anticuerpos contra VIH en personas infectadas o reacciones inespecíficas en muestras de personas no infectadas. Las mujeres embarazadas suelen tener reacciones inespecíficas sin estar infectadas. ${ }^{10}$

En los casos indeterminados se recomienda repetir la prueba después de un mes para confirmación. ${ }^{11}$ En el caso de que el resultado continúe indeterminado podría considerarse no infectada, ${ }^{12}$ excepto si hay exposición reciente a una pareja con VIH, que hace necesario conocer el estado serológico de la pareja. La gestante debe recibir el tratamiento hasta que se aclare el diagnóstico. Las pruebas de carga viral no son recomendadas para el diagnóstico ni para aclaración del caso indeterminado. ${ }^{13}$

En el caso que la mujer gestante no acceda al control prenatal, en el momento que sea captada, incluso en trabajo de parto, debe realizarse una prueba de tamizaje VIH para decidir conducta profiláctica en el caso que ésta sea reactiva; esta prueba es denominada "rápida", y tiene una sensibilidad similar a las pruebas "corrientes" ${ }^{14-16} \mathrm{La}$ falla en identificar la infección materna antes del parto resulta en un fracaso para la prevención de la transmisión madre-hijo de VIH.

En algunos casos se recomienda realizar la prueba de Elisa al recién nacido en las primeras 24 horas, para indicar la aplicación de la profilaxis postnatal. ${ }^{17}$ Los test rápidos se utilizan para definir la conducta profiláctica y deben ser con- firmados, cuando son reactivos, con el diagrama de flujo de diagnóstico existente: una prueba inmuno-enzimática y una prueba confirmatoria como Western Blot. La decisión de iniciar el esquema profiláctico considera el valor predictivo positivo de la prueba y el riesgo/beneficio de la terapia. ${ }^{18}$

El resultado de las pruebas negativas y positivas debe estar consignado en la historia clínica, lo que permite el seguimiento de cada gestante. Estos resultados deben ser informados a la paciente dentro de un proceso de asesoría post-prueba.

\section{CONTROL PRENATAL DE LA GESTANTE INFECTADA CON $\mathrm{VIIH}^{19-25}$}

El control prenatal de la embarazada infectada con VIH debe incluir:

- Información sobre VIH y embarazo y riesgo de transmisión de VIH de madre a hijo.

- Información sobre formas de prevención de la transmisión madre-hijo relacionadas con la gestación, parto y puerperio; que incluya los efectos de los medicamentos anti-retrovirales y sus efectos en el feto.

- Evaluación de antecedentes clínicos y factores de riesgo.

- Optimización del estado nutricional.

- Examen clínico completo que incluya cavidad orofaríngea y especuloscopia. Debe buscarse cervicitis muco-purulenta y úlceras genitales, y proveer el tratamiento.

- Clasificación del estadio de la enfermedad para establecer pronóstico y plan de manejo.

- Indicación a la gestante de su deber de informar al compañero sexual para su canalización a los servicios de asesoría, consejería y prueba voluntaria para VIH.

- Cuantificación de la carga viral de VIH, cada 3-4 meses o por lo menos en el momento de la detección y antes del parto.

- Recuento de linfocitos CD4+/CD8+, una vez por trimestre. 
- Tamizaje para sífilis, toxoplasmosis, citomegalovirus, hepatitis B (antígeno de superficie - AgHBs), hepatitis C y tuberculosis (PPD). El tamizaje óptimo debe realizarse en el primer y tercer trimestres.

- Realización de citología cérvico-vaginal, en lo posible con colposcopia. Repetición a las 8 semanas de la primera.

- Ecografía para determinar edad gestacional.

- Administración de la terapia anti-retroviral correspondiente con la consejería sobre los riesgos y beneficios de los medicamentos para el binomio madre-hijo.

- Monitoreo de la adherencia y las reacciones adversas a la terapia anti-retroviral.

- Seguimiento de pruebas de función renal y hepática.

- Profilaxis contra Mycobacterium tuberculosis (isoniacida y piridoxina), Pneumocystis carinii (trimetoprim sulfamethoxazol con CD $4<200 / \mathrm{mm}^{3}$ ), Mycobacterium avium complex (azitromicina con $\mathrm{CD} 4<50 / \mathrm{mm}^{3}$ ) y Toxoplasma gondii de acuerdo a los lineamientos para mujeres no embarazadas. ${ }^{26}$

- Evaluación del estado de inmunización. Evitar vacunas durante el embarazo, principalmente si se tienen cargas virales indetectables, pues pueden causar un rebote viral. ${ }^{27}$

- Indicación de consulta inmediata ante síntomas o signos relacionados con infecciones oportunistas.

- Indicación de consulta precoz ante síntomas de parto prematuro o ruptura prematura de membranas.

- Programación de la cesárea o indicaciones para el parto vaginal. Remisión para la atención al nivel correspondiente.

- Información sobre el uso y suministro de preservativos.

Son considerados factores asociados a la transmisión madre-hijo del VIH:

1. Disminución del recuento absoluto de linfocitos CD4 en la primera medición $\left(<200 \mathrm{~mm}^{3}\right){ }^{28}$

2. Carga viral: hay una asociación entre la carga viral y la probabilidad de transmisión sin tratamien- to, de $0 \%$ con $<1.000$ copias $/ \mathrm{mm}^{3}$ a $40,6 \%$ con $>100.000$ copias. ${ }^{28-31} \mathrm{La}$ carga viral predice el riesgo pero no el momento de la infección perinatal.

3. Uso de drogas intravenosas durante el embarazo.

4. SIDA avanzado.

5. Parto pretérmino.

6. Corioamnionitis. ${ }^{32}$

7. Presencia de antígeno p24 en el primer control o antes del parto.

8. Lactancia materna.

9. No suministro de ZDV mediante el protocolo PACTG 076. ${ }^{33}$

Los procedimientos diagnósticos prenatales “invasivos” están contraindicados, tales como amniocentesis, cordocentesis y biopsia de vellosidad corial.

\section{RECOMENDACIONES DE TERAPIA} ANTI-RETROVIRAL 19-25, 34, 35

Para la indicación de la terapia anti-retroviral en la gestante es importante recordar los cambios fisiológicos propios del embarazo como el tránsito gastrointestinal aumentado; el incremento del agua y la grasa corporal, del gasto cardíaco, la ventilación respiratoria, el flujo renal y hepático y de la reabsorción de sodio renal; la disminución de las proteínas plasmáticas y el cambio en las vías metabólicas; también, el transporte placentario de los medicamentos, la biotransformación placentaria y la excreción fetal.

En cualquiera de los escenarios que se presentan a continuación debe tenerse en cuenta que la terapia triple anti-retroviral permite disminuir la carga viral, la transmisión madre-hijo a menos de $2 \%$ y la aparición de resistencia (Watts) ${ }^{36}$; en lo posible, cualquiera de los esquemas debe usar zidovudina (ZDV) como fue establecido por el estudio Pediatrics AIDS Clinical Trials Group 076 (PACTG), no sólo por sus efectos en la reducción de la carga viral si no por sus resultados en la prevención de la transmisión madre hijo ${ }^{37}$ (Tabla 1). 
Tabla 1. Protocolo PACTG 076 de administración de zidovudina para prevenir transmisión madre a hijo de VIH

\section{Antes del parto}

ZDV 200 mg cada 8 horas VO, iniciar desde semana 14 de gestación y continuar hasta el comienzo del trabajo de parto (opción ZDV 300 mg cada 12 horas).

\section{Intraparto}

ZDV infusión de $2 \mathrm{mg} / \mathrm{kg}$ durante la primera hora y luego $1 \mathrm{mg} / \mathrm{kg} /$ hora hasta el parto.

\section{Al recién nacido}

ZDV suspensión $2 \mathrm{mg} / \mathrm{kg}$ cada seis horas VO; iniciar antes de las 48 horas de nacido.

Pretérmino 1,5 mg/kg IV ó 2 mg/kg VO cada 12 horas; si tenía más de 30 semanas al momento del parto, a las 2 semanas continuar cada 8 horas y si tenía menos de 30 semanas, a las 4 semanas continuar cada 8 horas.

Modificado de: Public Health Service Task Force. Recommendations for Use of Antiretroviral Drugs in Pregant HIV-1 Infected Women for Maternal Health and Interventions to Reduce Perinatal HIV- 1 Transmission in the United States. Disponible en http://AIDSinfo.nih.gov. Consultado 7 de enero de 2005.

Los análogos nucleósidos inhibidores de la transcriptasa reversa más usados son la zidovudina y la lamivudina; el análogo no nucleósido inhibidor de la transcriptasa reversa es la nevirapina y el inhibidor de la proteasa de mayor uso es el nelfinavir. ${ }^{38}$ Estos son los medicamentos con menos efectos adversos en madre e hijo.

Escenario 1. Mujeres embarazadas infectadas con VIH sin terapia anti-retroviral previa.

1. La mujer embarazada con VIH debe recibir evaluación clínica, inmunológica y virológica.

2. La selección e inicio de la terapia deben estar basadas en los parámetros usados en mujeres no embarazadas; sin embargo, los riesgos y beneficios conocidos y desconocidos de cada terapia durante el embarazo deben ser considerados y discutidos.

3. Los tres momentos de la profilaxis con ZDV (durante el embarazo, en el momento del parto y al neonato) deben tenerse en cuenta para todas las mujeres embarazadas infectadas con VIH así como para sus hijos o hijas, para disminuir el riesgo de transmisión perinatal.

4. La combinación de profilaxis con ZDV y otros medicamentos anti-retrovirales adicionales debe considerarse en mujeres con carga viral mayor de 1.000 copias $/ \mathrm{mm}^{3}$ y según estado clínico e inmunológico.

5. Para gestantes con cargas virales inferiores a 1.000 copias $/ \mathrm{mm}^{3}$ puede considerarse la monoterapia con $\mathrm{ZDV}$, en sus tres momentos. ${ }^{39}$

6. Las mujeres que están en el primer trimestre del embarazo pueden retardar el inicio de la terapia hasta después de la semana 10 a 14 de gestación.

7. En pacientes que recibieron ZDV por menos de diez semanas antes del parto, puede utilizar nevirapina, la cual disminuye del 6 al 2\% la posibilidad de transmisión. ${ }^{24}$

Escenario 2. Mujeres infectadas con VIH que recibían terapia anti-retroviral previa al embarazo.

1. Mujeres infectadas que reciben terapia anti-retroviral y cuyo embarazo es diagnosticado después del primer trimestre pueden continuar la terapia previa.

2. Mujeres que reciben tratamiento anti-retroviral y se diagnostica embarazo durante el primer trimestre. Se debe evitar el uso de efavirenz o delavirdina en el primer trimestre de embarazo.

3. Informar a la paciente sobre los riesgos para el feto de los medicamentos que se están empleando al igual que los beneficios de continuarlos, y luego tomar la decisión.

4. La ZDV debe ser un componente esencial en el tratamiento anti-retroviral prenatal tan pronto como sea posible. También debe aplicarse endovenoso en el momento del parto y suministrarse vía oral al neonato por seis semanas.

5. De ser necesaria la suspensión del tratamiento se puede reiniciar después de la semana 14. Esta estrategia puede producir rebote viral y aumento del riesgo de transmisión. Se recomienda suspender y reiniciar los tres medicamentos al mismo tiempo, excepto los inhibidores no nucleósidos de la trans- 
criptasa reversa que deben ser suspendidos 7 días antes que los otros anti-retrovirales.

6. Los casos de resistencia comprobada al tratamiento anti-retroviral se manejan igual que en mujeres no embarazadas.

7. Los casos de infección aguda, falla virológica o supresión subóptima del virus después del tratamiento se manejan igual que en las mujeres no embarazadas con casos idénticos.

8. La nevirapina no está indicada en monodosis antes del parto y para el recién nacido. ${ }^{35}$

Escenario 3. Mujeres infectadas con VIH, en trabajo de parto, que no han recibido previamente terapia anti-retroviral.

Están disponibles varios regímenes terapéuticos que incluyen:

1. Una dosis de nevirapina vía oral (200 mg) a la embarazada al inicio del trabajo de parto y una dosis de nevirapina al recién nacido (2 mg/kg/dosis) en las primeras 48-72 horas de vida.

2. Zidovudina y lamivudina (3TC) durante el trabajo de parto, seguida de una semana de ZDV3TC al recién nacido. ${ }^{39-42}$

3. ZDV endovenoso a la madre, al menos, tres horas antes del parto y vía oral por seis semanas al recién nacido.ZDV en el recién nacido debe iniciarse en las primeras 48 horas de vida. ${ }^{43}$

4. Combinación del esquema 1 con el $3 .^{44}$

Escenario 4. Recién nacidos de madres que no han recibido tratamiento anti-retroviral durante el embarazo o intraparto.

1. Se informará a la madre sobre la opción de iniciar ZDV o ZDV y 3TC por seis semanas. El tratamiento debe iniciarse a las seis-doce horas de nacido.

2. Algunos médicos recomiendan ZDV y la asociación con otros medicamentos anti-retrovirales si hay sospecha de resistencia viral al ZDV. Sin embargo, se desconoce la eficacia para prevenir la transmisión y no está definida la eficacia para el recién nacido. En estos casos especiales, es respon- sabilidad del personal médico ofrecer esta opción de manejo al neonato.

Escenario 5. Recién nacido de madre a quien no se realizó prueba de VIH.

1. Se debe realizar una prueba rápida (resultado en máximo dos horas) a la madre o al niño para decidir conducta profiláctica.

2. Si la prueba es reactiva se debe iniciar tratamiento con ZDV o ZDV+3TC y realizar prueba confirmatoria con Western Blot.

3. Si la prueba confirmatoria es negativa suspender los medicamentos.

4. Si la prueba confirmatoria es positiva continuar por seis semanas.

En estudios realizados en animales, la profilaxis iniciada 24 a 36 horas después del nacimiento es ineficaz para prevenir la transmisión. ${ }^{45} \mathrm{La}$ infección generalmente se establece entre la primera y segunda semana de edad, por lo que iniciar la profilaxis después de las 48 horas de vida puede ser inoficioso.

\section{RESISTENCIA A}

\section{ANTI-RETROVIRALES 19-25, 34-35}

Todas las mujeres embarazadas deben recibir terapia anti-retroviral altamente efectiva para reducir la replicación viral, el riesgo de transmisión perinatal, mejorar el estado inmunológico y el riesgo de resistencia viral.

En mujeres con menos de 1.000 copias $/ \mathrm{mm}^{3}$, la terapia anti-retroviral combinada es opcional y la madre debe conocer la posibilidad de resistencia al ZDV o 3TC.

La resistencia más común es registrada con el uso de monoterapias con nevirapina o de biterapias con ZDV+3TC, situación que debe ser suficientemente evaluada de acuerdo a los escenarios puestos en consideración.

Si la paciente tiene antecedente de resistencia al ZDV, y el esquema de tratamiento no lo incluye, en el momento del parto se aplica ZDV endovenoso y se suministra $Z D V$ vía oral al recién nacido. 
En mujeres con tratamiento combinado, que por alguna razón deban interrumpirlo durante el embarazo, se suspenderá toda la medicación y el reinicio se hará con todas. Cuando el tratamiento incluye nevirapina, debe suspenderse ésta primero y continuar con los otros dos medicamentos por 7 días más, para disminuir la posibilidad de resistencia.

Las recomendaciones para el manejo de la embarazada con resistencia al tratamiento son iguales que para la paciente no embarazada. La adherencia al tratamiento es importante para evitar la resistencia.

Las pruebas de resistencia a los anti-retrovirales tienen las mismas indicaciones que en mujeres no embarazadas: infección aguda, rebrote viral o viremia persistente, en un paciente que recibe terapia anti-retroviral combinada.

\section{RECOMENDACIONES PARA EL PARTO Y PROFILAXIS DEL RECIÉN NACIDO EXPUIESTO ${ }^{19-25,34-35}$}

Las recomendaciones durante el parto van dirigidas a decidir sobre la realización de la cesárea, la prevención del parto pretérmino, la reducción del tiempo entre la ruptura prematura de membranas y el parto a menos de 4 horas y a minimizar la exposición fetal a la sangre materna.

En todos los casos se deben ajustar las decisiones según las condiciones de cada paciente, sin embargo, con una carga viral reciente mayor de 1.000 copias puede estar indicada la cesárea, por la evidencia de la disminución de la probabilidad de transmisión durante el parto. ${ }^{46-49}$ Por esta razón se recomienda realizar una carga viral a las 34-36 semanas de gestación.

Cuando las cargas virales son menores de 1.000 copias $/ \mathrm{mm}^{3}$ y la gestante recibe tratamiento, no ha sido posible determinar la utilidad de la cesárea en comparación con el parto vaginal, dada la probabilidad de transmisión con esta carga viral, menor de $2 \%$.

Es de resaltar que en mujeres no infectadas con VIH, el riesgo de complicaciones y muertes postcesárea es 6 a 7 veces mayor que post-parto vaginal; ${ }^{50}$ sin embargo datos recientes, en mujeres infectadas con VIH, no identificaron un riesgo mayor. ${ }^{51,52}$ Para disminuir este riesgo se recomienda el tratamiento de la vaginosis o la cervico-vaginitis bacteriana o bien, el uso de antibióticos profilácticos.

En la decisión de realizar una cesárea se debe considerar el acceso, la adherencia y el tipo de terapia anti-retroviral, el antecedente de control prenatal adecuado, eficacia en la disminución de la carga viral y edad gestacional a la captación. ${ }^{53}$

La programación de la cesárea debe hacerse para la semana 38, para disminuir la posibilidad de inicio de trabajo de parto o de ruptura prematura de membranas, aunque debe preverse un aumento en el riesgo de dificultad respiratoria en el recién nacido.

En el caso que se opte por parto vaginal, de todas maneras se debe asegurar la aplicación endovenosa de ZDV, o tan pronto se diagnostique ruptura prematura de membranas.

Deben evitarse la amniotomía, uso de electrodos en el cuero cabelludo fetal, toma de muestras de sangre de cuero cabelludo fetal, uso de instrumentos para asistir el parto (espátulas o fórceps) y otros procedimientos que causen trauma en el niño. La no realización de episiotomía podría disminuir la exposición del niño a la sangre materna. La ruptura prematura de membranas de más de 4 horas de evolución, dobla el riesgo de transmisión intra-parto. ${ }^{54}$

El midazolam y preparaciones con ergotamina no deben usarse en pacientes que reciben inhibidores de la proteasa (nelfinavir) o efavirenz, por interferencia entre medicamentos.

El niño debe ser limpiado y bañado antes de aplizar inyecciones (p.ej. vitamina $\mathrm{K}$ ), tomar muestras o realizar otro tipo de procedimiento invasivo.

Escenario 1. Mujer infectada con VIH que consulta en el tercer trimestre (más de 36 semanas), no ha recibido tratamiento. Tiene pendiente reporte de carga viral y recuento de linfocitos.

1. Inicio inmediato de tratamiento anti-retroviral, incluir al menos ZDV y seguir el protocolo PCTG 076. 
2. Recomendación de cesárea programada a las 38 semanas, según evaluación clínica.

3. Información a la paciente sobre los riesgos de la cesárea, incluyendo la posibilidad de infecciones asociadas.

4. No dar leche materna al recién nacido.

5. Según el reporte de carga viral y recuento de linfocitos CD4/CD8 se definirá el tratamiento a la madre.

Escenario 2. Mujer infectada con VIH que inicia control prenatal temprano en el primer trimestre y recibe tratamiento anti-retroviral completo. Tiene más de 1.000 copias en la carga viral y 36 semanas de embarazo.

1. Continuar tratamiento hasta que disminuya el número de copias.

2. Programación de cesárea a las 38 semanas.

3. Información a la paciente sobre los riesgos de la cesárea, incluyendo la posibilidad de infecciones asociadas.

4. ZDV endovenoso al menos tres horas antes de la cesárea.

5. Continuar los otros medicamentos, incluso durante el parto y postparto.

6. ZDV al recién nacido por seis semanas.

7. No dar leche materna al recién nacido.

8. Enfatizar a la paciente la importancia de la adherencia al tratamiento.

Escenario 3. Mujer infectada con VIH, tratamiento completo anti-retroviral y niveles indetectables de carga viral, con 36 semanas de gestación.

1. Información a la paciente sobre la probabilidad de infección perinatal menor de 2\% con estos niveles de carga viral, incluso con parto vaginal.

2. Información a la paciente sobre los riesgos de la cesárea, incluyendo la posibilidad de infecciones asociadas.

3. ZDV endovenoso al menos tres horas antes de la cesárea.

4. ZDV al recién nacido por seis semanas.
5. No dar leche materna al recién nacido.

Escenario 4. Mujer infectada con el VIH que tiene cesárea programada y presenta ruptura de membranas y trabajo de parto.

1. Iniciar inmediatamente ZDV endovenoso.

2. Si el trabajo de parto evoluciona rápidamente se continuará el parto vaginal.

3. Si la ruptura prematura de membranas ocurrió antes de la semana 32, debe hacerse manejo conservador con terapia anti-retroviral continuada, intentar prolongación del embarazo para reducir el riesgo de complicaciones por prematurez.

4. Si el trabajo de parto se calcula con retardo y el cuello uterino está en dilatación mínima, aplicación de ZDV endovenoso y realización de cesárea.

5. Mínima manipulación y procedimientos invasivos.

6. El recién nacido es tratado por seis semanas con ZDV.

7. No dar leche materna al recién nacido.

\section{RECOMENDACIONES PARA LA} LACTANCIA Y EL PUIERPERIO ${ }^{19-25,34-35, ~} 55$

La lactancia materna exclusiva tiene una probabilidad de transmisión de VIH postparto de 9,3\% a los 18 meses de seguimiento, ${ }^{56}$ por lo que deben darse indicaciones sobre alimentación con fórmula láctea exclusiva hasta los 6 meses de vida del niño, sin olvidar el control de la galactorrea. En el caso de las mujeres y los recién nacidos que tuvieron diagnóstico tardío con prueba rápida debe considerarse la posibilidad de reiniciar la lactancia materna si la prueba confirmatoria es negativa.

Son factores de riesgo para la transmisión mediante la lactancia materna: la carga viral en la leche materna, mastitis aguda o subclínica, abscesos en mama y sero-conversión durante la lactancia. ${ }^{57} \mathrm{La}$ alimentación mixta se constituye también en un importante factor de riesgo. ${ }^{58-60}$

El suministro de leche de fórmula debe ser continuo y su preparación debe tener las condiciones óptimas de higiene y limpieza para facilitar la 
práctica de la alimentación. Esta condición debe ser valorada integralmente, procurando educar en las alternativas de alimentación. ${ }^{61}$

En el postparto inmediato la paciente debe tener una adecuada evaluación virológica (carga viral), inmunológica (CD4/CD8) y de su estado clínico, para ofrecer el mejor tratamiento.

Durante el puerperio se debe brindar asesoría acerca del acceso y adherencia al tratamiento, teniendo en cuenta la pérdida de interés tras la prevención de la transmisión madre-hijo de VIH, o la depresión postparto. La asesoría consta de información sobre el curso de la infección, medidas de control, cuidados al niño, pruebas diagnósticas y de seguimiento, y toxicidad potencial de los medicamentos. Esto es fortalecido mediante la búsqueda del soporte social y familiar para la mujer en el postparto.

También requiere asesoría para planificación familiar, que incluya la posibilidad de ligadura y sección de trompas. Los anticonceptivos orales que contienen estradiol, mantienen niveles más bajos en sangre cuando la mujer recibe nevirapina, nelfinavir, ritonavir o amprenavir. El dispositivo intrauterino no se recomienda por el riesgo de otras infecciones de transmisión sexual.

Si la madre no está recibiendo ayuda debe ser remitida a la entidad que se encargará de su manejo. Deben buscar otros problemas como no afiliación al sistema de salud, drogadicción, desempleo, violencia doméstica, etc, y realizar la orientación necesaria.

\section{SEGUIMIENTO DEL NIÑO EXPUIESTO AL VIH ${ }^{19-25,34-35}$}

En el recién nacido es inútil la determinación de anticuerpos contra VIH, mediante Elisa o Western Blot debido al paso de anticuerpos maternos al feto que determinan que el resultado sea positivo. Se deben realizar pruebas virales directas como PCRARN, PCR-ADN y cultivo viral. ${ }^{1-3,16}$

\section{Reacción en cadena de la polimerasa} para ADN de VIH-1. Prueba que tiene una sensibilidad de 96\% y una especificidad del 99\% en el primer mes. ${ }^{62}$ Esta es la prueba recomendada para el tamizaje inicial. El valor predictivo positivo en el primer mes es de 55,8\% y después del primer mes, $83,2 \%{ }^{63}$

\section{Reacción en cadena de polimerasa para} ARN de VIH-1. Tiene buena sensibilidad y especificidad; no se afecta cuando ha recibido sólo ZDV, pero no se conoce su efectividad diagnóstica cuando se ha recibido más de un medicamento. Es menos sensible y específica que PCR-ADN. ${ }^{64}$

3. Cultivos de VIH-1. Son costosos, demorados (14-28 días), difíciles y han sido reemplazados por los exámenes anteriores.

4. Antígeno p24. No se recomienda por su menor sensibilidad y mayor tasa de falsos positivos.

La sangre se obtiene del neonato (no del cordón por el alto riesgo de falsos positivos). Si el neonato no ha recibido leche materna, el b-ADN idealmente se debe tomar al nacimiento, a las 4-7 semanas y a las 16 semanas. Si dos pruebas son negativas, se descarta infección con VIH-1. Si las tres cargas virales son negativas, el valor predictivo positivo es cercano a $95 \%{ }^{66}$

Si una prueba de carga viral sale positiva se repite de inmediato; si se confirma que el niño está infectado, se debe iniciar tratamiento en forma temprana. Esto es válido para todos los menores de doce meses sin importar su estado clínico o inmunológico, ni la carga viral.

A los doce meses se solicita la prueba de Elisa para descartar que los anticuerpos de la madre estén presentes; si es negativa y tenemos una prueba negativa de carga viral, se descarta infección; si es positiva se repite a los 18 meses, si es negativa se descarta infección, si es positiva, hay infección. ${ }^{67}$

En el momento del parto se verificará la información sobre la posibilidad de otras coinfecciones como tuberculosis, hepatitis B, hepatitis C, sífilis, toxoplasmosis, citomegalovirus o herpes. El manejo y tratamiento del neonato dependerá de los datos de la madre o el resultado de los exámenes practicados. ${ }^{21} \mathrm{Si}$ se sospecha infección materna con VIH-2, se realizarán exámenes específicos. 
Para el seguimiento del neonato se debe tener en cuenta:

1. PCR-ADN en las primeras 48 horas, semanas 4-6 y 16.

2. ELISA para VIH a los 12 y 18 meses.

3. Cuadro hemático-VSG: al nacimiento y mensualmente (1-6 meses)

4. CD4/CD8: al mes y tres meses.

5. ZDV hasta las seis semanas.

6. Profilaxis para Pneumocystis jiroveci posterior a la profilaxis con ZDV (semana 6). Suspensión con descarte de infección con VIH.

7. Valoración de TBC de la madre, en el caso de detección de TB hematógena, se procede a realizar diagnóstico en el recién nacido. En el caso de otro tipo de TB, practicar aislamiento respiratorio de la madre.

8. Vacunación según esquema del Programa Ampliado de Inmunizaciones. La aplicación de BCG puede requerir definir la infección en el niño. ${ }^{68}$

10. Vacunación complementaria con anti-neumococo heptavalente, anti-varicela y anti-influenza según esquema y valoración de recuento de CD4.

El padre y los hermanos del niño expuesto deben ser valorados con pruebas de Elisa para VIH. La edad no es impedimento para realizar la prueba, ya que incluso en adolescentes se ha hecho evidente la enfermedad, después de ser infectados por transmisión vertical.

\section{VIGILANCIA DE LA TOXICIDAD POR MEDICAMENTOS ${ }^{19-25,34-35}$}

La información sobre la toxicidad de los medicamentos proviene generalmente de estudios de toxicidad en animales, experiencias anecdóticas, registro y ensayos clínicos.

\section{Toxicidad en gestantes}

Los análogos nucleósidos inhibidores de la transcriptasa tienen hasta $0,5 \%$ de riesgo de producir toxicidad mitocondrial por la interferencia en la replicación mitocondrial; sin embargo el riesgo es menor cuando se usa ZDV o 3TC. Esta afectación puede llevar a síndrome HELLP y acidosis láctica con esteatosis.

Los síntomas relacionados con los efectos adversos de la nevirapina incluyen fatiga, malestar general, anorexia, náusea e ictericia; hasta en 4\% de los casos, hay aumento de las transaminasas relacionado con rash. Su presencia puede incrementar el riesgo de toxicidad hepática entre 3,2 y 7,3 veces. Cuando el CD4 es mayor de 250 células/mm3 ese riesgo aumenta a 9,8 veces. ${ }^{69}$ Este efecto adverso sucede en las primeras 18 semanas de tratamiento, por lo que es recomendado el monitoreo de la función hepática cada dos semanas por los primeros 4 meses de tratamiento.

El nelfinavir ocasiona hiperglicemia, facilita la aparición o exacerbación de la diabetes mellitus y puede producir cetoacidosis, incluso aumenta el riesgo de hiperglicemia asociada con el embarazo.

En general, deben evitarse los medicamentos anti-retrovirales durante el primer trimestre del embarazo, en especial, efavirenz, hidroxiúrea, didanosina, indinavir o imprenavir.

Los estudios con terapia triple anti-retroviral no han registrado diferencias en la presentación de parto pretérmino, bajo peso al nacer o APGAR disminuido. ${ }^{69}$

\section{Toxicidad in útero o postparto}

El daño en el feto depende del medicamento, edad gestacional del feto en el momento de la exposición, duración de la exposición, interacción con otros agentes cuando el feto está expuesto y la base genética.

La ZDV puede producir anemia en el recién nacido, alcanzando un pico máximo a las 6 semanas y resolviendo a la semana 12 sin tratamiento. Por esta razón se debe hacer control de hemoglobina y hematocrito al nacimiento, uno y dos meses después; existe posibilidad de disfunción mitocondrial $(0,46 \%)$ y de convulsiones o enfermedad neurológica $(1,1 \%)$ pero son riesgos menores en comparación con los beneficios de evitar la transmisión perinatal. 
Es recomendable el seguimiento al menos por 18 meses de los niños, tanto para su diagnóstico como para la valoración de los efectos adversos y la resistencia anti-retroviral (en el caso de los infectados).

\section{NOTA INFORMATIVA}

En Colombia, desde 2003, se desarrolla el Proyecto Nacional de Reducción de la Transmisión Madre Hijo de VIH por una alianza de Onusida-Colombia, Instituto Nacional de Salud y la Federación Colombiana de Cooperativas de Hospitales, con financiación de la Comisión Europea. Este proyecto facilita los componentes e insumos básicos de atención de la madre infectada con VIH y su hijo expuesto. Su objetivo es promover el reconocimiento del derecho y el acceso a la prueba de VIH a la gestante no afiliada al Sistema General de Seguridad Social en Salud y apoyar a los servicios de salud en la atención de la gestante y al recién nacido.

Información sobre el proyecto: línea de atención gratuita 018000110073, PBX 1-6164676, correo electrónico: proyectomadrehijo@yahoo.com.

\section{REFERENCIAS}

1. Onusida. Informe sobre la epidemia mundial de SIDA 2004. Cuarto informe mundial. Ginebra: Onusida; 2004.

2. Steinbrok R. The AIDS epidemic in 2004. N Engl J Med 2004;351:115-7.

3. Prieto F. Veinte años del VIH en Colombia, 19832003. Datos de la vigilancia epidemiológica. Inf Quinc Epidem Nac 2003;8:355-64.

4. Garcia-Bernal R, Klaskala W, Castro J, Zhang G, Baum M. VIH/AIDS epidemic in Colombia: regional differences in epidemic trends. AIDS 1997;11:1297-8.

5. Mock PA, Shaffer N, Bhadrakom C, Siriwasin W, Chotpitayasunondh T, Chearskul S, et al. Maternal viral load and timing of mother-to-child HIV transmission, Bangkok, Thailand. Bangkok Collaborative Perinatal HIV Transmission Study Group. AIDS 1999;13:407-14.

6. Fowler MG, Simonds RJ, Roongpisuthipong A. Update on perinatal HIV transmission.Pediatr Clin North Am 2000;47:21-38.
7. Institute of Medicine, National Research Council. Reducing the Odds: Preventing Perinatal Transmission of HIV in the United States. Washington, DC: National Academy Press; 1999.

8. Ministerio de Salud (Colombia). Resolución 00412 de 2000. Por la cual se establecen las actividades, procedimientos e intervenciones de demanda inducida y obligatorio cumplimiento y se adoptan las normas técnicas y guías de atención para el desarrollo de las acciones de protección específica y detección temprana y la atención de enfermedades de interés en salud pública. Guías de atención No. 11: VIH/sida.

9. Centers for Disease Control and Prevention. Revised guidelines for HIV counseling, testing, and referral. MMWR Recomm Rep 2001;50:1-57.

10. Gwinn M, Redus MA, Granade TC, Hannon WH, George JR. HIV-1 serologic test results for one million newborn dried-blood specimens: assay performance and implications for screening. J Acquir Immune Defic Syndr 1992;5:505-12.

11. Celum CL, Coombs RW, Lafferty W, Inui TS, Louie $\mathrm{PH}$, Gates CA, et al. Indeterminate human immunodeficiency virus type 1 Western blots: seroconversion risk, specificity of supplemental tests, and an algorithm for evaluation. J Infect Dis 1991;164:656-64.

12. Jackson JB, MacDonald KL, Cadwell J, Sullivan C, Kline WE, Hanson $\mathrm{M}$ et al. Absence of HIV infection in blood donors with indeterminate Western blot tests for antibody to HIV-1. N Engl J Med 1990;322:217-22.

13. Centers for Disease Control and Prevention. Revised recommendations for HIV screening of pregnant women. MMWR Recomm Rep 2001;50:63-85.

14. Minkoff H, O'Sullivan MJ. The case for rapid HIV testing during labor. JAMA 1998; 279:1743-4.

15. Grobman WA, Garcia PM. The cost-effectiveness of voluntary intrapartum rapid human immunodeficiency virus testing for women without adequate prenatal care. Am J Obstet Gynecol 1999;181:1062-71.

16. Kane B. Rapid testing for HIV: why so fast? Ann Intern Med 1999;131:481-3.

17. King SM; American Academy of Pediatrics Committee on Pediatric AIDS; American Academy of Pediatrics Infectious Diseases and Immunization Committee. Evaluation and treatment of the human immunodeficiency virus 1 - exposed infant. Pediatrics 2004;114:497-505.

18. Mofenson LM, Munderi P. Safety of antiretroviral prophylaxis of perinatal transmission for HIV-infected 
pregnant women and their infants. J Acquir Immune Defic Syndr 2002;30:200-15.

19. Watts DH. Management of human immunodeficiency virus infection in pregnancy. N Engl J Med 2002;346:1879-91.

20. Krist AH. Obstetric care in patients with HIV disease. Am Fam Physician 2001;63:107-16, 121-2.

21. Mofenson LM, Committee on Pediatric AIDS. Technical Report: perinatal human immunodeficiency virus testing and prevention of transmission. Pediatrics 2000;106:e88.

22. Krist AH, Crawford-Faucher A. Management of newborns exposed to maternal HIV infection. Am Fam Physician 2002;65:2049-56.

23. Watts DH. Managament of human immunodeficiency virus Infection in pregnancy. $\mathrm{N}$ Engl J Med 2002;346:1879-91.

24. Brocklehurst P, Volmink J. Antiretrovirals for reducing the risk of mother-to-child transmission of HIV infection. Cochrane Database Syst Rev 2002;(2): CD003510.

25. Public Health Service Task Force. Recommendations for Use of Antiretroviral Drugs in Pregnant HIV-1 Infected Women for Maternal Health and Interventions to Reduce Perinatal HIV-1 Transmission in the United States. Disponible en: http://AIDSinfo.nih.gov. Consultado 7 de enero de 2005.

26. U.S. Public Health Service (USPHS) and Infectious Diseases Society of America (IDSA). 1999USPHS/IDSA Guidelines for the prevention of opportunistic infectio$\mathrm{ns}$ in persons infected with human immunodeficiency virus. MMWR Recomm Rep 1999;48:1-59, 61-6.

27. Moss W, Clements J, Halsey N. Immunization of children at risk of infection with Human Immunodeficiency Virus. Bull World Health Organ 2003;81:61-70.

28. Mofenson LM, Lambert JS, Stiehm ER, Bethel J, Meyer WA 3rd, Whitehouse J, et al. Risk factors for perinatal transmission of human immunodeficiency virus type 1 in women treated with zidovudine. Pediatric AIDS Clinical Trials Group Study 185 team. N Engl J Med 1999;341:385-93.

29. Garcia PM, Kalish LA, Pitt J, Minkoff H, Quinn TC, Burchett SK, et al. Maternal levels of plasma human immunodeficiency virus type 1 RNA and the risk of perinatal transmission. Women and Infants Transmission Study Group. N Engl J Med 1999;341:394402 .
30. Thea DM, Steketee RW, Pliner V, Bornschlegel K, Brown T, Orloff S, et al. The effect of maternal viral load on the risk of perinatal transmission of HIV-1. New York City Perinatal HIV Transmission Collaborative Study Group. AIDS 1997;11:437-44.

31. Mayaux MJ, Dussaix E, Isopet J, Rekacewicz C, Mandelbrot L, Ciraru-Vigneron N, et al. Maternal virus load during pregnancy and mother-to-child transmission of human immunodeficiency virus type 1: the French perinatal cohort studies. J Infect Dis 1997;175:172-5.

32. Van Dyke RB, Korber BT, Popek E, Macken C, Widmayer SM, Bardeguez A, et al. The Ariel Project: a prospective cohort study of maternal-child transmission of human immunodeficiency virus type 1 in the era of maternal antiretroviral therapy. J Infect Dis 1999;179:319-28.

33. Harris NS, Thompson SJ, Ball R, Hussey J, Sy F. Zidovudine and perinatal human immunodeficiency virus type 1 transmission: a population-based approach. Pediatrics 2002;109(4):e60.

34. King SM, American Academy of Pediatrics Committee on Pediatric AIDS; American Academy of Pediatric Infectious Diseases and Immunization Committee. Evaluation and treatment of the human immunodeficiency virus-1-exposed infant. Pediatrics 2004;114:497-505.

35. Connor EM, Sperling RS, Gelber R, Kiselev P, Scott G, O'Sullivan MJ, et al. Reduction of maternal-infant transmission of human immunodeficiency virus type 1 with zidovudine treatment. N Engl J Med 1994;331:1173-80.

36. Cooper ER, Charurat M, Mofenson L, Hanson IC, Pitt J, Diaz C, et al. Combination anti-retroviral strategies for the treatment of pregnant HIV-1 infected women and prevention of perinatal HIV-1 transmission. J Acquir Immune Defic Syndr 2002;29:484-94.

37. Sperling RS, Shapiro DE, Coombs RW, et al. Maternal viral load, zidovudine treatment, and the risk of transmission of human immunodeficiency virus type 1 from mother to infant. Pediatric AIDS Clinical Trials Group Protocol 076 Study Group. N Engl J Med 1996; 335:1621-9.

38. Talaie H, Nava-Ocampo AA, Koren G. Antiretroviral treatment of maternal HIV infection. Can Fam Physician 2004;50:865-8.

39. Ioannidis JP, Abrams EJ, Ammann A, Bulterys M, Goedert JJ, Gray L, et al. Perinatal transmission of human immunodeficiency virus type 1 by pregnant women with RNA virus loads $<1000$ copies/ml. J Infect Dis 2001;183:539-45. 
40. Guay LA, Musoke P, Fleming T, Bagenda D, Allen M, Nakabiito $C$, et al. Intrapartum and neonatal single dosis nevirapine compared with zidovudine for prevention of mother to child transmission of HIV-1 in Kampala, Uganda : HIVNET 012 randomised trial. Lancet 1999;354:795-802.

41. Jourdain G, Ngo-Giang-Huong N, Le Coeur S, Bowonwatanuwong C, Kantipong P, Leechanachai P, et al. Intrapartum exposure to nevirapine and subsequent maternal responses to nevirapine-based antiretroviral therapy. N Engl J Med 2004;351:229-40.

42. The Petra Study Team. Efficacy of three short-course regimens of zidovudine and lamivudine in preventing early and late transmission of HIV-1 from mother to child in Tanzania, South Africa, and Uganda (Petra study); a randomised double-blind placebo-controlled trial. Lancet 2002;359:1178-86.

43. Wade NA, Birkhead GS, Warren BL, Charbonneau TT, French PT, Wang L, et al. Abbreviated regimens of zidovudine prophylaxis and perinatal transmission of the human immunodeficiency virus. N Engl J Med 1998;339:1409-14.

44. Dorenbaum A, Cunningham CK, Gelber RD, Culnane M, Mofenson L, Britto P, et al. Two-dose intrapartum/ newborn nevirapine and standard antiretroviral therapy to reduce perinatal HIV-1 transmission: a randomized trial. JAMA 2002;288:289-98.

45. Van Rompay KK, Otysula MG, Marthas ML, Miller CJ, McChesney MB, Pedersen NC. Inmediate zidovudine treatments protects simian immunodeficiency virus-infected newborn macaques against rapid onset of AIDS. Antimicrob Agents Chemother 1995;39:125-31.

46. Dunn DT, NewellML, Mayaux MJ, Kind C, Hutto C, Goedert JJ, et al. Mode of delivery and vertical transmission of HIV-1: a review of prospective studies. Perinatal AIDS Collaborative Transmission Studies. J Acquir Immune Defic Syndr 1994;7:1064-6.

47. Kuhn L, Bobat R, Coutsouidis A, Moodley D, Coovadia HM, Tsai WY, et al. Cesarean deliveries and maternalinfant HIV transmission: results from a prospective study in South Africa. J Acquir Immune Defic Syndr Hum Retrovirol 1996;11:478-83.

48. The Internacional Perinatal HIV Group. The mode of delivery and the risk of vertical transmission of human immunodeficiency virus type 1: a meta-analysis of 15 prospective cohort studies. N Engl J Med 1999;340:977-87.
49. The European Mode of Delivery Collaboration. Elective caesarean-section versus vaginal delivery in prevention of vertical HIV-1 transmission: a randomised clinical trial. Lancet 1999;353:1035-9.

50. van Ham MA, van Dongen PW, Mulder J. Maternal consequences of caesarean section: a retrospective study of intra-operative and post-operative maternal complications of caesarean section during a 10-year-period. Eur J Obstet Gynecol Reprod Biol 1997;74:1-6.

51. Read J, Tuomala R, Kpamegan E, Zorrilla C, Landesman S, Brown G, et al. Mode of delivery and postpartum morbidity among HIV infected women: The Women and Infants Transmission Study (WITS). J Acquir Immune Defic Syndr 2001;26:236-45.

52. Rodriguez EJ, Spann C, Jamieson D, Lindsay M. Postoperative morbidity associated with caesarean delivery among human immunodeficiency virusseropositive women. Am J Obstet Gynecol 2001; 184:1108-11.

53. Landers DV, Duarte G. Mode of delivery and the risk of vertical transmission of HIV-1. N Engl J Med 1999;341:205.

54. Landesman SH, Kalish LA, Burns DN, Minkoff H, Fox $\mathrm{HE}$, Zorrilla C, et al. Obstetrical factors and the transmission of human immunodeficiency virus type 1 from mother to child. The Women and Infants Transmission Study. N Engl J Med 1996;334:1617-23.

55. Bulterys M, Fowler MG, Van Rompay KK, Kourtis AP. Prevention of mother-to-child transmission of HIV-1 through breast feeding: past, present, and future. J Infect Dis 2004;189:2149-53.

56. Breastfeeding and HIV International Transmission Study Group. Late postnatal transmission of HIV-1 in breast-fed children: an individual patient data metaanalysis. J Infect Dis 2004;189:2154-66.

57. Embree JE, Njenga S, Datta P, Nagelkerke NJ, Ndinya-Achola JO, Mohammed Z, et al. Risk factors for postnatal mother-child transmission of HIV-1. AIDS 2000;14:2535-41.

58. Coutsouides A, Pillay K, Kuhn L, Spooner E, Tsai WY, Coovadia HM; South African Vitamin A Study Group. Methods of feeding and transmission of HIV-1 from mothers to children by 15 months of age : prospective cohort study from Durban, South Africa. AIDS 2001;15:379-87.

59. Rollins N, Meda N, Becquet R, Coutsoudis A, Humphrey J, Jeffrey B, et al. Preventing postnatal transmission of HIV-1 through breastfeeding: mo- 
difying infant feeding practices. J Acquir Immune Defic Syndr 2004;35:188-95.

60. Kiarie JN, Richardson BA, Mbori-Ngacha D, Nduati RW, John-Stewart GC. Infant feeding practices of women in a perinatal HIV-1 prevention study in Nairobi, Kenya. J Acquir Immune Defic Syndr 2004;35:75-81.

61. Read JS; American Academy of Pediatrics Committee on Pediatric AIDS. Human milk, breastfeeding, and the transmission of human immunodeficiency virus type 1 in the United States. Pediatrics 2003;112:1196-205.

62. Dunn DT, Brand CD, Krivine A, et al. The sensitivity of HIV-1 DNA polymerase chain reaction in the neonatal period and the relative contributions of intra-uterine and intra-partum transmission. AIDS 1995;9:F7:11.

63. Owens DK, Holodniy M, McDonadl TW, Scout J, Sonnad S. A meta-analytic evaluation of the polymerase chain reaction for the diagnosis of HIV infection in infants. JAMA 1996;275:1342-8.

64. Steketee RW, Abrams EJ, Thea DM, Brown TM, Lambert G, Orloff S, et al. Early detection of perinatal human immunodeficiency virus (HIV) type 1 infection using HIV RNA amplification and detection. New York City Perinatal HIV Transmission Colaborative Study. J Infec Dis 1997;175:707-11.

65. Rouet F, Montcho C, Rouzioux C, Leroy V, Msellati P, Kottan JB, et al. ANRS. Early diagnosis of pediatric HIV-1 Infection among African breast-fed children using quantitative plasma HIV RNA assay. AIDS 2001;15:1849-56.
66. American Academy of Pediatrics. Comittee on Pediatric AIDS. Evaluation and medical treatment of the HIVexposed infant. Pediatrics 1997;99:909-17.

67. Centers for Disease Control and Prevention. Guidelines for nacional human immunodeficiency virus case surveillance, including monitoring for human immunodeficiency virus infection and acquired immunodeficiency syndrome. MMWR Recomm Rep 1999;48:1-27, 2931.

68. Atkinson WL, Pickering LK, Schwartz B, Weniger BG, Iskander JK, Watson JC; Centers for Disease Control and Prevention. General Recommendations on Immunization: Recommendations of the Advisory Comittee on Immunization Practices (ACIP) and the American Academy of Family Physicians (AAFP). MMWR Recomm Rep 2002;51:1-35.

69. Stern JO, Robinson PA, Love J, Lanes S, Imperiale MS, Mayers DL. A comprehensive hepatic safety analysis of nevirapine in different populations of HIV infected patients. J Acquir Immune Defic Syndr 2003;34:S2133.

70. Tuomala RE, Shapiro DE, Mofenson LM, Bryson Y, Culnane M, Hughes MD, et al. Antiretroviral therapy during pregnancy and the risk of an adverse outcome. N Engl J Med 2002;346:1863-70. 